\title{
Longitudinale Assoziationen zwischen depressiven Symptomen und Typ-2-Diabetes sowie deren Auswirkung auf die Mortalität von Hausarztpatienten
}

\author{
L. Pieper ${ }^{1}$, J. Dirmaier ${ }^{2}$, J. Klotsche ${ }^{1}$, C. Thurau ${ }^{1}$, D. Pittrow ${ }^{3}$, H. Lehnert ${ }^{4}$, W. März ${ }^{5}$, U. \\ Koch $^{2}$, H.-U. Wittchen ${ }^{1}$ \\ 1 Institut für Klinische Psychologie und Psychotherapie, Technische Universität Dresden, Dresden \\ 2 Institut und Poliklinik für Medizinische Psychologie, Universitätsklinikum Hamburg-Eppendorf, Hamburg- \\ Eppendorf \\ 3 Institut für Klinische Pharmakologie, Technische Universität Dresden, Dresden \\ 4 Medizinische Klinik, Universitätsklinikum Schleswig-Holstein, Campus Lübeck, Lübeck \\ 5 Synlab, Heidelberg
}

\section{Zusammenfassung}

Es gibt widersprüchliche Befunde darüber, ob depressive Symptome Risikofaktoren für die Neumanifestation eines Diabetes sind oder ob umgekehrt auch Diabetes ein Risikofaktor für depressive Zustände ist. Daher untersuchen wir die längsschnittlichen wechselseitigen Assoziationen zwischen depressiven Symptomen und Typ-2-Diabetes (T2D) sowie die Auswirkungen des gemeinsamen Auftretens beider Erkrankungen auf die Mortalität anhand einer Stichprobe von Hausarztpatienten im Verlauf eines im Mittel 3,5-jährigen Beobachtungszeitraums. Die depressive Symptomatik wurde anhand des Depression Screening Questionnaire (DSQ) kategorial sowie dimensional betrachtet. Die Einteilung in Patienten mit normalem Nüchternblutzucker (NBZ), erhöhtem NBZ sowie T2D (unbehandelt, medikamentös, mit Insulin/kombiniert behandelt) erfolgte nach Arztangaben beziehungsweise nach Laborbefunden zur Baseline-Untersuchung. Die Inzidenz des T2D bei Patienten mit beziehungsweise ohne depressive Symptome betrug 25,6 und 20,9 pro 1000 Personenjahre. Bei dimensionaler Betrachtung des DSQ erhöhte sich das T2D-Risiko (unadjustiert) um das 1,03-Fache [KI (95\%): 1,01-1,06] bei punktweisem Anstieg des DSQ. Die Inzidenz depressiver Symptome per 1000 Personenjahre betrug 30,5 für Patienten mit normalem, 34,2 für Patienten mit erhöhtem NBZ, 36,4 für unbehandelte, 32,3 für oral behandelte und 47,8 für insulinbehandelte T2D-Patienten. Verglichen mit Patienten mit einem normalen NBZ hatten insulinbehandelte Patienten ein höheres Risiko für depressive Symptome [HR: 1,71; KI (95\%): 1,03-2,83] und oral behandelte T2D-Patienten ein niedrigeres Risiko [HR: 0,58; KI (95\%): 0,36-0,96]. Verglichen mit Patienten ohne T2D und depressiver Symptomatik ist das Vorliegen beider Erkrankungen mit einer erhöhten Mortalität assoziiert [HR: 2,49; KI (95\%):1,45-4,28]. Die Ergebnisse deuten an, dass vor allem eine Insulinbehandlung bei T2DPatienten mit inzidenten depressiven Symptomen assoziiert ist.

Schlüsselwörter: Depressive Symptomatik, Typ-2-Diabetes, Mortalität, Längsschnittuntersuchung, Primärärztliche Versorgung 


\title{
Longitudinal associations between depressive symptoms and type 2 diabetes and their impact on mortality in primary care patients
}

\begin{abstract}
It is unclear whether depressive symptoms are a risk factor for incident diabetes or diabetes is a risk factor for depressive conditions. Therefore, we examined the longitudinal bidirectional associations between depressive symptoms and type 2 diabetes (T2D) as well as the impact of both diseases on (all cause) mortality in a sample of primary care patients over a 3.5-years follow-up period on average. Depressive symptomatology, defined by the Depression Screening Questionnaire (DSQ), was examined both categorically and dimensionally. Patients were categorized as normal fasting glucose (NFG), impaired fasting glucose (IFG), and T2D (untreated, oral antidiabetics, insulin/combined treatment) according to physician ratings and baseline lab values. Incidence rates of T2D were 25.6 and 20.9 per 1000 person-years for those with and without depressive symptoms, respectively. The unadjusted risk of incident type 2 diabetes was 1.03 times higher (CI(95\%): 1.01-1.06) for each 1point increment in DSQ score. The incidence rates of elevated depressive symptoms per 1000 personyears were 30.5 for NFG, 34.2 for IFG, 36.4 for untreated T2D, 32.3 for oral treated T2D, and 47.8 for insulin/combined-treated T2D patients. Compared to NFG patients, insulin-treated patients had a higher risk of incident depressive symptoms (HR: 1.71; CI(95\%): 1.03-2.83) and oral-treated patients had a lower risk (HR: 0.58; CI(95\%): 0.36-0.96). Higher mortality rates were associated with both diseases compared to patients without T2D or depressive symptoms at baseline (HR: 2.49; $\mathrm{CI}(95 \%): 1.45-4.28)$. Results indicate that especially insulin treatment in T2D patients is associated with incident depressive symptoms.
\end{abstract}

Keywords: Depressive symptoms, Type 2 diabetes mellitus, Mortality, Longitudinal design, Primary care

Die Prävalenz klinisch manifester Depressionen und depressiver Symptome ist bei DiabetesTyp-2 (T2D)-Patienten höher als in der Allgemeinbevölkerung [1, 2]. Die querschnittlichen Assoziationen zwischen beiden Krankheitsbildern können unterschiedliche Ursachen haben. Die depressive Symptomatik kann der T2D-Erkrankung vorausgehen und ihre Entstehung begünstigen. Umgekehrt kann der T2D die Entstehung depressiver Symptome entscheidend beeinflussen. Mit Blick auf den zeitlichen Zusammenhang zwischen beiden Erkrankungen fanden Golden et al. [3], dass Personen mit depressiver Symptomatik ein moderat erhöhtes Risiko für die Entwicklung eines T2D haben. Diese Assoziation war nach Adjustierung von soziodemografischen, ökonomischen und metabolischen Faktoren signifikant, jedoch nicht nach einer Adjustierung von Lebensstilfaktoren. Diese Ergebnisse stehen im Einklang mit einer Serie nachfolgender Untersuchungen, die eine positive Assoziation zwischen depressiven Störungen und T2D berichten [4, 5, 6, 7]. Faktoren, die diese Assoziation beeinflussen, sind unter anderem der Bildungsstatus [8], ein ungesunder Lebensstil (Bewegungsmangel, Übergewicht) [9], Veränderungen im neuroendokrinen System [10] und Entzündungsreaktionen [11, 12]. Zum Teil werden diese Faktoren als Erklärungsmodelle herangezogen. Es gibt jedoch auch Studien (wie beispielsweise [13]), die keine erhöhten Diabetesraten infolge depressiver Störungen feststellen konnten.

Als weiteres Ergebnis stellten Golden et al. [3] heraus, dass bei Patienten ohne depressive Symptome zur Baseline, die einen behandelten T2D hatten, das Risiko für die Ausbildung depressiver Symptome erhöht war. Diese Assoziation war unabhängig vom Body Mass Index (BMI), sozioökonomischen Status und Komorbiditäten. Auch für diese Assoziation finden sich weitere quer- [14] und längsschnittliche Befunde in der Literatur [1, 15, 16]. Die Heterogenität der Forschungslage zeigt sich in Studien [17, 18], die eine negative Assoziation 
zwischen Insulinresistenz und Übergewicht (als Diabetesvorstufen) und inzidenten depressiven Störungen herausstellten. Auch bei Golden et al. [3] waren ein erhöhter Blutzuckerspiegel und ein unbehandelter T2D mit einem niedrigeren Risiko für depressive Symptome assoziiert. Als Risikofaktoren für depressive Störungen bei Diabetespatienten gelten die folgenden diabetesunabhängigen Faktoren: weibliches Geschlecht, Beziehungsstatus (alleinstehend, geschieden, verwitwet), jüngeres Lebensalter und niedriger sozioökonomischer Status. Als diabetesabhängige Faktoren wurden Folgekomplikationen, Insulintherapie, Hypoglykämien und ein hoher HbA1c bestätigt [14, 19].

Unabhängig von der Art des Zusammenhangs zwischen depressiver Symptomatik und T2D berichteten zahlreiche Untersuchungen über die negativen Auswirkungen des gemeinsamen Auftretens dieser Erkrankungen auf die Entwicklung von Folgekomplikationen [20, 21] und die Mortalitätsraten [21, 22] der Patienten.

\section{Fragestellungen}

Vor diesem Hintergrund ergaben sich für diese Untersuchung folgende Fragestellungen: (1) Sind bei Hausarztpatienten depressive Symptome mit dem Neuauftreten von T2D assoziiert?

(2) Ist im Vergleich zu Patienten mit normalem Nüchternblutzucker (NBZ) ein unbehandelter oder behandelter (mit und ohne Insulin) T2D mit inzidenten depressiven Symptomen assoziiert? (3) Wie wirkt sich das Auftreten depressiver Symptome und von T2D auf die Mortalität von Hausarztpatienten aus?

\section{Methoden}

\section{Die DETECT-Studie}

Die Beantwortung der oben genannten Fragen erfolgte mittels Daten der DETECT-Studie. DETECT ist eine querschnittliche und prospektiv längsschnittliche klinisch epidemiologische Studie in der primärärztlichen Versorgung in Deutschland. Ziel war die Bestimmung der Häufigkeit von Herz-Kreislauf-Erkrankungen, Diabetes und mit diesen Erkrankungen assoziierten Faktoren. Im Jahr 2003 nahmen 3188 Hausärzte in ganz Deutschland an der Studie teil und dokumentierten mittels eines Fragebogens den Gesundheitszustand von über 55.500 Patienten. Zusätzlich füllte jeder Patient einen Fragebogen aus. Eine Teilstichprobe von 851 Ärzten nahm zusätzlich an der Laborkomponente der DETECT-Studie teil und entnahm Blutproben von insgesamt 7519 zufällig ausgewählten Patienten. Diese Patienten bildeten die Grundgesamtheit für den längsschnittlichen Teil der DETECT-Studie. Sie wurden im Jahr 2004 (erstes Follow-up) und im Jahr 2007/8 (zweites Follow-up) mit der gleichen Methodik wie zur Baseline nachuntersucht. Insgesamt konnten von 6826 Patienten Followup-Informationen erhoben werden. Eine ausführliche Methoden- und Designbeschreibung der Studie wurde bereits an anderer Stelle publiziert $[23,24]$ und findet sich auch unter http://www.detect-studie.de. Die DETECT-Studie wurde von der Ethikkommission der TU Dresden befürwortet (AZ:EK149092003). Alle Prozeduren bei der Datenerhebung und Auswertung folgten den Regeln der Deklaration von Helsinki. Alle Patienten wurden umfassend über die Untersuchung aufgeklärt und unterzeichneten eine Einverständniserklärung. 


\section{Instrumente und Erhebungsmethoden}

\section{Erfassung der depressiven Symptomatik}

Die depressive Symptomatik wurde in allen drei Untersuchungswellen im Patientenfragebogen mit dem DSQ (Depression Screening Questionnaire) erhoben [25]. Mit diesem Fragebogen können depressive Störungen im Sinne einer Major Depression erfasst werden. Er besteht aus zehn Symptomfragen, die auf den Kriterien des DSM-IV sowie den Forschungskriterien des ICD-10 basieren. Diese Items beziehen sich auf das Befinden und Verhalten in den letzten zwei Wochen und werden mithilfe einer dreistufigen Likert-Skala (,,an den meisten Tagen“ $=2$, ,einige Tage“ $=1$, ,nie“ $=0$ Punkte) beantwortet. Für die Bestimmung der depressiven Symptomatik wurde in den Analysen ein DSQ-Summen-Score von $\geq 8$ als Cut-off herangezogen (minimaler Wert $0=$ keine depressive Symptomatik; maximaler Wert 20). In unserer kategorialen Betrachtung konnten also Patienten mit einem Wert $\geq 8$ verschiedene Ausprägungsgrade einer Depression aufweisen (milde bis moderate depressive Zustände sowie auch schwere Depressionen). Die Interne Konsistenz des DSQ beträgt 0,83 (Cronbachs Alpha). Die Retest-Reliabilität beträgt 0,82 [25,26]. Es wurden in der DETECT-Baseline-Erhebung keine Fragen zur antidepressiven Medikation gestellt. Daher beruht die Erhebung der depressiven Symptomatik ausschließlich auf den Angaben der Patienten im DSQ. Neu auftretende (inzidente) depressive Symptome wurden bei Patienten angenommen, die zur Baseline-Untersuchung einen DSQ $<8$ hatten, aber zu einer der beiden Follow-up-Untersuchungen einen Wert von mindestens 8 berichteten.

\section{Erhebung des Diabetes-Status}

Die Diagnose T2D basiert auf den klinischen Urteilen der Ärzte am Studientag, den Angaben zur Diabetesmedikation und den Ergebnissen der laborchemischen Untersuchung (NBZ). Diese Aspekte wurden in allen drei Untersuchungswellen der DETECT-Studie erhoben. Es wurden keine weiteren Kriterien zur Diagnosestellung, zum Beispiel aus Leitlinien, vorgegeben, um die Diagnose- und Behandlungssituation in der primärärztlichen Versorgung nicht zu verfälschen. Die Zuordnung des Diabetestyps erfolgte durch die behandelnden Ärzte. Bei fehlenden Angaben zum Diabetestyp wurden diese durch Angaben aus den späteren Untersuchungswellen ergänzt [27]. Der NBZ wurde bei den Patienten, die zum Zeitpunkt der Blutentnahme seit mindestens acht Stunden keine Nahrung zu sich genommen hatten aus den Blutproben bestimmt (standardisierte Auswertung in einem Zentrallabor). Bei den restlichen Patienten wurden die vom Arzt dokumentierten NBZ-Werte aus der Patientenakte herangezogen. Für die vorliegende Untersuchung wurden die Patienten unterteilt in Personengruppen mit normalem $(<100 \mathrm{mg} / \mathrm{dl})$ und erhöhtem NBZ $(100-125 \mathrm{mg} / \mathrm{dl})$ sowie Personengruppen mit vom Arzt diagnostiziertem T2D, die entweder keine antidiabetische Medikation erhielten, ausschließlich oral oder mit Insulin (auch in Kombination mit oralen Antidiabetika) behandelt wurden. Ein inzidenter T2D wurde bei den Patienten diagnostiziert, die die Kriterien eines T2D zwar nicht zur Baseline, aber zu einer der beiden Follow-upUntersuchungen erfüllten.

\section{Erfassung der Kontrollvariablen}

Die Angaben zum Alter, Geschlecht, zur Anzahl der absolvierten Schuljahre, zum beruflichen Status (berufstätig, Hausfrau, Arbeitslos, Rentner) und Familienstand (ledig, verheiratet, geschieden/verwitwet) stammen aus dem Patientenbogen der Baseline-Untersuchung (2003). Daraus stammen auch die Angaben zu Problemen mit gesunder Ernährung sowie zur körperlichen Aktivität (mehr oder weniger als zwei Stunden körperliche Betätigung/Woche). Der Rauchstatus (nie, früher, aktuell) wurde im Arztfragebogen dokumentiert. Die Compliance wurde mit mehreren Items sowohl im Arzt- als auch im Patientenbogen erfasst. Diese unterschiedlichen Informationsquellen wurden zu zwei Compliance-Scores verrechnet 
(Medikamenten-Compliance sowie Behavioral Compliance). Blutdruck und Taillenumfang wurden von den Ärzten standardisiert (nach schriftlichen Instruktionen durch das Studienzentrum) gemessen und dokumentiert. Laborwerte [Insulin, HbA1c, HDL- und LDLCholesterin, Triglyzeride und C-reaktives-Protein (CRP)] wurden aus den Blutproben der Patienten in einem Zentrallabor standardisiert bestimmt. Die genauen Laborprozeduren sowie Messmethoden und Interassay-Koeffizienten wurden bereits an anderer Stelle detailliert beschrieben [23].

\section{Erfassung der Mortalität}

Die Mortalität wurde von den Ärzten in den Follow-up-Untersuchungen dokumentiert (Todeszeitpunkt und Ursache). Zudem wurden die Ärzte gebeten, den Totenschein zu übermitteln. Für diese Untersuchung erfolgte keine Unterteilung in unterschiedliche Todesursachen, und die Mortalität wurde unabhängig von der Ursache berichtet.

\section{Analysevorgehen}

Aufgrund der drei unterschiedlichen Zielstellungen dieser Untersuchung sind drei unterschiedliche Stichproben erforderlich. In der Ergebnisdarstellung wird bei jeder der drei Analysen auf die gleiche Weise vorgegangen. Im ersten Schritt werden die BaselineCharakteristika der Untersuchungsgruppen (nach Depressivitätsstatus beziehungsweise nach Diabetesstatus) miteinander verglichen. Dafür wurden F-Tests für kontinuierliche Variablen und Chi2-Tests für kategoriale Variablen eingesetzt. Diskrete Survival-Modelle wurden für das Auftreten eines inzidenten T2D (Analyse 1) und inzidenter depressiver Symptome (Analyse 2) mit definierten Zeitpunkten zum Einjahres- und Fünfjahres-Follow-up geschätzt. Hazard-Ratios wurden mit komplementären Log-log-Regressionsmodellen [28, 29] im Personen-X-Follow-up-Zeit-Datensatz (Anzahl der Zeilen in Daten pro Person entsprechen Anzahl der Jahre unter Risiko) geschätzt. Die Schätzung der Hazard-Ratios für die Mortalität (Analyse 3) erfolgte mittels Cox-Regression [30]. Die Standardfehler der Modellparameter wurden mit der Huber-White-Sandwich-Matrix robust geschätzt [31]. Ein zweiseitiger p-Wert von $<0,05$ wurde als Signifikanzgrenze angenommen. Alle statistischen Analysen wurden mit Stata 11.0 [32] durchgeführt.

Analyse 1 - depressive Symptome und inzidenter T2D. Für diese Analyse wurden Patienten mit einem prävalenten T2D zur Baseline $(\mathrm{N}=1213)$ sowie Patienten ohne BaselineInformationen zur depressiven Symptomatik $(\mathrm{N}=193)$ ausgeschlossen. Damit ergab sich eine Grundgesamtheit von 5420 Patienten. T2D-Inzidenzraten wurden für Patienten mit depressiver Symptomatik berechnet und mit den Raten bei Patienten ohne depressive Symptome verglichen. Zusätzlich wurde der DSQ-Score dimensional betrachtet und das Hazard-Ratio (HR) für einen punktweisen Anstieg des DSQ-Scores angegeben. Um die Mechanismen aufzudecken, die den Zusammenhang zwischen erhöhter depressiver Symptomatik und T2D erklären, wurden folgende Strategien angewandt: Modell 1 betrachtet den Zusammenhang unadjustiert, Modell 2 schließt Alter und Geschlecht in die Analyse ein. Modell 3 adjustiert zusätzlich nach Taillenumfang. Die Modelle 4 bis 8 schließen die Aspekte aus Modell 2 sowie folgende Variablen ein: Modell 4, metabolische Variablen (Lipidwerte, Blutdruck, Insulin); Modell 5, Entzündungsmarker (CRP); Modell 6, sozioökonomische Variablen (Schuljahre, Berufsstand); Modell 7, Lebensstilvariablen (Rauchstatus, körperliche Aktivität, Alkoholkonsum, Ernährungsprobleme); Modell 8, Compliance. Modell 9 adjustiert nach allen Variablen aus allen Modellen.

Analyse 2 - T2D und inzidente depressive Symptome. Patienten, die zum Zeitpunkt der Baseline-Untersuchung einen DSQ-Score von $\geq 8$ Punkten hatten, wurden aus dieser Analyse ausgeschlossen $(\mathrm{N}=1091)$. Dies gilt auch für Patienten, von denen keine Informationen aus 
dem DSQ (N=424) oder keine Follow-up-Informationen zum Diabetes-Status vorlagen $(\mathrm{N}=30)$. Daraus ergab sich für die Analyse eine Grundgesamtheit von 5281 Patienten. Die kumulative Inzidenz depressiver Symptome wurde für Patienten mit NBZ-Werten im normalen Bereich $(<100 \mathrm{mg} / \mathrm{dl})$ berechnet und mit der Inzidenz bei Patienten mit erhöhten NBZ-Werten sowie bei T2D-Patienten (unbehandelt, medikamentös und mit Insulin behandelt) verglichen. Es wurde wiederum eine Reihe von Modellen berechnet, um die Mechanismen zu untersuchen, die den Zusammenhang zwischen T2D-Status und erhöhter depressiver Symptomatik erklären. Das Basismodell ist identisch zu dem in Analyse 1. Die Folgemodelle umfassen die Faktoren Taillenumfang, sozioökonomische und Lebensstilfaktoren, Compliance sowie Lipide, Blutdruck und HbA1c.

Analyse 3 - T2D depressive Symptome und Mortalität. Aus dieser Analyse wurden Patienten ausgeschlossen, über die zum Zeitpunkt der Baseline-Untersuchung keine Informationen zum Diabetes-Status oder zum DSQ vorlagen $(\mathrm{N}=265)$. Daraus ergab sich eine Analysestichprobe von $\mathrm{N}=6561$. Es wurde die Mortalität für Patienten ohne depressive Symptome und T2D zur Baseline bestimmt und mit den Mortalitätsraten von Patienten mit depressiven Symptomen, mit T2D oder mit beiden Erkrankungen verglichen. Auch bei Analyse 3 wurde eine Reihe von Modellen mit möglichen Einflussfaktoren auf die Mortalität berechnet. Es wurde mit einem unadjustierten Modell begonnen und im Folgenden nach Alter und Geschlecht adjustiert. $\mathrm{Zu}$ diesem Modell wurden in weiteren Analysen weitere Faktoren hinzugezogen (analog zu Analyse 2).

\section{Ergebnisse}

Analyse 1 - Depressive Symptomatik und inzidenter T2D

Verglichen mit Hausarztpatienten ohne depressive Symptomatik zur Baseline (DSQ-Score $<8$ Punkten) waren Patienten mit depressiver Symptomatik (DSQ $\geq 8$ ) etwas älter, häufiger männlich, hatten eine kürzere Schulbildung und waren seltener berufstätig (häufiger arbeitslos, berentet oder Hausfrau, . Tab. 1). Sie waren seltener verheiratet, dafür häufiger geschieden oder verwitwet. Bezüglich der Lebensstilfaktoren waren Patienten mit einem DSQ $\geq 8$ häufiger aktuell Raucher, seltener körperlich aktiv und berichteten häufiger Ernährungsprobleme. Sie hatten höhere CRP-Werte und einen höheren Taillenumfang.

Uni- und multivariate Analysen. In der Gruppe der Patienten mit einem DSQ unter 8 Punkten traten insgesamt 320 inzidente T2D-Fälle auf. Bei den Patienten mit einem DSQ $\geq 8$ waren es 53 Fälle. Die Inzidenzrate über im Mittel 3,2 Jahre betrug 25,6 pro 1000 Personenjahre bei Personen mit erhöhter depressiver Symptomatik und 20,9 für diejenigen ohne erhöhte depressive Symptomatik [HR=1,24, KI (95\%): 0,93-1,66].

Beim Vergleich der Patienten mit einem DSQ $<8$ und DSQ $\geq 8$ zur Baseline konnten weder im unadjustierten (Modell 1, . Tab. 2) noch in einem der adjustierten Modelle (Modelle 2 bis 8) signifikante HRs für die Inzidenz eines T2D berichtet werden. Bei dimensionaler Betrachtung des DSQ im Hinblick auf einen inzidenten T2D wurden - wenn auch in geringer Ausprägung - signifikante HRs gefunden [1,03; KI (95\%), 1,01-1,06, unadjustiert]. Diese Assoziationen blieben bei Adjustierung nach Alter, Geschlecht, metabolischen und inflammatorischen Markern sowie Lebensstilfaktoren signifikant. Bei Adjustierungen nach Taillenumfang, sozioökonomischen Faktoren, Compliance sowie allen Faktoren verlor die Assoziation ihre Signifikanz. 
Verglichen mit Patienten mit normalem NBZ-Spiegel waren diejenigen mit erhöhten NBZWerten oder einem unbehandelten oder behandelten T2D älter, häufiger männlich, hatten eine kürzere Schulbildung, waren seltener berufstätig, häufiger ledig oder geschieden/verwitwet, hatten häufiger früher geraucht (seltener aktuell) und niedrigere Compliance-Werte (. Tab. 3). Sie hatten ebenfalls niedrigere HDL-Spiegel, höhere Triglyzerid- und Insulin-Werte, einen höheren Taillenumfang und häufiger Bluthochdruck.

Uni- und multivariate Analysen. Bei Patienten mit normalen NBZ-Werten zur Baseline traten insgesamt 342 Fälle mit inzidenten depressiven Symptomen auf (mittlerer Follow-upZeitraum: 3,3 Jahre). Bei erhöhten NBZ-Werten waren es 89 inzidente Fälle, 54 bei unbehandelten T2D-Patienten, 46 bei oral behandelten T2D-Patienten und 39 bei Insulin/kombiniert behandelten T2D-Patienten. Das entspricht einer Inzidenzrate für depressive Symptome von 30,7 pro 1000 Personenjahre bei Patienten mit normalem Glukosespiegel, von 34,4 bei erhöhten NBZ-Werten, von 34,8 bei unbehandelten T2DPatienten, von 32,3 bei oral therapierten T2D-Patienten und von 47,8 bei Insulin/kombiniert behandelten T2D-Patienten. Verglichen mit Hausarztpatienten mit normalen Blutzuckerspiegeln wurden nur für Insulin/kombiniert behandelte T2D-Patienten signifikant höhere HRs für inzidente depressive Symptome gefunden [1,57; KI (95\%): 1,12-2,18] (. Tab. 4). Die Assoziation blieb auch bei Adjustierung nach Alter, Geschlecht, Taillenumfang, Lebensstilfaktoren und Compliance signifikant, verschwand jedoch bei Adjustierung nach sozioökonomischen Faktoren, Lipiden, Blutdruck, HbA1c und im volladjustierten Modell. Unbehandelte T2D-Patienten sowie Patienten mit erhöhtem NBZ unterschieden sich nicht signifikant von Patienten mit normalem NBZ bezüglich des Risikos für inzidente depressive Störungen. Bei oral behandelten Patienten verminderte sich die Häufigkeit inzidenter depressiver Störungen nach Adjustierung der Compliance [0,58; KI (95\%): 0,36-0,96].

\section{Analyse 3: Depressive Symptomatik, T2D und Mortalität}

Verglichen mit Patienten ohne T2D und ohne depressive Symptome zur Baseline waren Patienten mit depressiver Symptomatik, mit T2D oder beiden Erkrankungen älter, hatten eine kürzere Schulbildung, waren häufiger berentet, seltener verheiratet und häufiger geschieden/verwitwet (. Tab. 5). Sie hatten häufiger Probleme, sich gesund zu ernähren, waren häufiger noncompliant und gaben an, sich weniger körperlich zu betätigen. Vor allem Patienten mit T2D oder beiden Erkrankungen waren häufiger weiblich, hatten häufiger Hypertonie und einen höheren Taillenumfang.

Uni- und multivariate Analysen. Von den Patienten ohne T2D und depressive Symptomatik zur Baseline waren 123 Personen im Follow-up-Zeitraum verstorben (mittlerer Follow-upZeitraum: 3,2 Jahre). In der Patientengruppe mit depressiven Symptomen zur Baseline waren es 41, unter den T2D-Patienten 76 und in der Gruppe von Patienten mit beiden Erkrankungen 16. Daraus ergab sich eine Mortalitätsrate pro 1000 Personenjahre von 8,0 bei Patienten ohne T2D und depressive Symptomatik, von 19,8 bei Patienten mit depressiven Symptomen ohne T2D, von 23,3 bei Patienten mit T2D ohne depressive Symptomatik und von 31,6 pro 1000 Personenjahre bei Patienten mit beiden Erkrankungen zur Baseline. Sowohl unadjustiert als auch im voll adjustierten Modell (. Tab. 6) waren die HRs für Mortalität sowohl in der Patientengruppe mit depressiven Symptomen [zum Beispiel in Modell 2: 2,36; KI (95\%): 1,65-3,38] als auch in der T2D-Patientengruppe [Modell 2: 1,64; KI (95\%): 1,23-2,19] gegenüber der Gruppe ohne diese Erkrankungen signifikant erhöht. Die höchsten HRs wurden 
bei Patienten, bei denen depressive Symptomen und T2D gleichzeitig vorlagen, zur Baseline gefunden [Modell 2: 2,49; KI (95\%): 1,45-4,28].

\section{Diskussion}

In der longitudinalen Analyse bestätigt die DETECT-Studie die wechselseitige Assoziation zwischen depressiven Symptomen und T2D (allerdings nicht unabhängig von Kovariablen) sowie ein erhöhtes Risiko für vorzeitiges Versterben bei Vorliegen einer oder beider Erkrankungen.

\section{Einfluss depressiver Symptome auf inzidenten T2D}

Bei dimensionaler Betrachtung (Anstieg um einen Punkt des DSQ-Scores) waren depressive Symptome zur Baseline nur schwach mit inzidentem T2D assoziiert. Bei kategorialer Betrachtung der depressiven Symptomatik konnte kein statistisch signifikanter Zusammenhang herausgestellt werden. Die Assoziationen sind im Vergleich zu Golden et al. [3] insgesamt schwächer ausgeprägt. Die bei dimensionaler Betrachtung gefundenen Assoziationen waren unabhängig von Alter, Geschlecht, metabolischen, inflammatorischen und Lebensstilfaktoren, blieben jedoch nach Kontrolle von sozioökonomischem Status, Compliance sowie im komplett adjustierten Modell nicht länger signifikant. Die schwach ausgeprägten Zusammenhänge zwischen depressiver Symptomatik und inzidentem T2D deuten darauf hin, dass die depressive Symptomatik eher vermittelnd wirkt und mit etablierten Risikofaktoren (Übergewicht, falsche Ernährung und Bewegungsmangel [9], metabolisches Syndrom) [33] konfundiert und damit eher einen indirekten Einfluss auf die Entwicklung eines Diabetes hat [34]. So fanden beispielsweise Mezuk et al. [8], dass die Entwicklung eines Diabetes bei Patienten mit Depressionen stark vom Bildungsstatus abhängig ist. Weitere Untersuchungen belegen, dass eine Aktivierung des neuroendokrinen Systems [10] sowie Entzündungsreaktionen und Insulinresistenz [11, 12] die Entwicklung eines T2D begünstigen können. Depressive Störungen stehen wiederum eng mit diesen Prozessen in Verbindung [21, 35].

\section{Einfluss des Diabetesstatus auf inzidente depressive Symptome}

Ein mit Insulin oder kombiniert behandelter T2D war positiv mit inzidenten depressiven Symptomen assoziiert. Keine signifikanten longitudinalen Assoziationen wurden im Vergleich zu Patienten mit normalem NBZ, bei Patienten mit erhöhten NBZ-Konzentrationen und bei unbehandelten T2D-Patienten gefunden. Bei Adjustierung nach Alter, Geschlecht, Taillenumfang und Compliance war ausschließlich ein oral behandelter T2D mit einer niedrigeren inzidenten depressiven Symptomatik assoziiert. Bei Golden et al. [3] wurde keine Unterscheidung zwischen oral und mit Insulin behandelten Diabetespatienten vorgenommen. Die Autoren fanden in der Gruppe der behandelten T2D-Patienten positive Assoziationen zu inzidenten depressiven Symptomen. Bei den unbehandelten Diabetespatienten wurden keine signifikanten Assoziationen nachgewiesen, und bei den Patienten mit erhöhtem NBZ fanden sich positive Assoziationen mit einer niedrigeren depressiven Symptomatik. Die Autoren folgerten daraus, dass vor allem der psychische Stress beim Umgang mit der Diabeteserkrankung zu erhöhten Depressionsraten führt. Auch in dieser Untersuchung scheint nicht der Diabetes selbst für die häufig in der Literatur beschriebenen Assoziationen [2, 14] zwischen Diabetes und inzidenten depressiven Störungen hauptverantwortlich zu sein, sondern eher die Insulintherapie und die damit verbundenen Komplikationen und Lebenseinschränkungen. Eine andere Studie [16] berichtet, dass die Assoziation zwischen 
T2D und inzidenten depressiven Symptomen nach Kontrolle von komorbiden Erkrankungen abgeschwächt wurde. Andere Untersuchungen zeigten sogar negative Assoziationen zwischen Insulinresistenz, Übergewicht und milderen T2D-Verläufen und inzidenten depressiven Störungen $[17,18]$. Auch die in dieser Untersuchung unter bestimmten Bedingungen (Modell 7) gefundene negative Assoziation zwischen T2D und depressiven Symptomen spricht für ein komplexes Beziehungsgeflecht zwischen beiden Erkrankungen und verschiedenen weiteren Risikofaktoren.

\section{Einfluss depressiver Symptome und von T2D auf die Mortalität}

Im Vergleich zu Patienten ohne depressive Symptome und ohne T2D zur Baseline haben einzeln betrachtet sowohl Patienten mit depressiven Symptomen als auch mit T2D ein erhöhtes Mortalitätsrisiko. Das Mortalitätsrisiko steigt nochmals an, wenn beide Faktoren gleichzeitig auftreten. Dieses Ergebnis steht in Einklang mit den Befunden anderer Studien $[21,22]$, die ebenfalls über ein erhöhtes Mortalitätsrisiko bei Patienten mit Diabetes und depressiver Symptomatik berichten. Nicht nur das Mortalitäts-, sondern auch das Morbiditätsrisiko ist bei Vorliegen beider Erkrankungen erhöht [21, 36]. So haben frühere Studien gezeigt, dass depressive Symptome mit dem Auftreten von Nephropathien [37], Retinopathien [38, 39], Neuropathien [40, 41] und makrovaskulären Erkrankungen [42, 43] assoziiert sind. Depressionen wirken sich aufgrund der damit assoziierten ungesünderen Lebensweise (wenig körperliche Aktivität, ungesunde Ernährung) bei Diabetespatienten negativ auf die Behandlung [44] aus. Weitere Studien berichten über negative Auswirkungen depressiver Störungen auf die Blutzuckerselbstkontrolle und Compliance [45] sowie auf die Stoffwechsellage (gemessen über den HbA1c) von T2D-Patienten [46]. Neben einer erhöhten Mortalität verursachen depressive Störungen bei Diabetespatienten eine höhere subjektiv berichtete Krankheitslast [47], eine eingeschränkte Lebensqualität [36, 47, 48] sowie gehäuft Einschränkungen im Arbeitsleben [49].

\section{Stärken der Studie}

Die DETECT-Studie erlaubt als eine der wenigen Studien die longitudinale Untersuchung des Zusammenhangs zwischen depressiver Symptomatik und Diabetes. Da beide Erkrankungen zu jedem der drei Untersuchungszeitpunkte erhoben wurden, ist bei derselben Grundgesamtheit die Betrachtung der Assoziationen in beide Richtungen möglich. Durch die detaillierte Erhebung des diabetischen Status (Arztdiagnose, Laborwerte, Therapie) sowie einer Vielzahl an Kontrollvariablen sind eine Abgrenzung zum Typ-1-Diabetes sowie eine sehr differenzierte Betrachtung verschiedener Stoffwechselzustände möglich. DETECT stellt eine für Deutschland repräsentative Zufallsauswahl von Hausärzten und ihren Patienten dar. Die Ergebnisse sind damit auf den primärärztlichen Versorgungssektor in Deutschland verallgemeinerbar.

\section{Limitationen der Studie}

Die Erfassung der depressiven Symptomatik wurde mittels eines Patientenfragebogens und nicht über ein klinisch strukturiertes Interview realisiert. Die Gabe von Antidepressiva wurde nicht erfasst. Es wurde auch keine Unterscheidung zwischen uni- und bipolaren depressiven Störungen vorgenommen. Der DSQ erfasst die depressive Symptomatik über zwei Wochen. Daher ist es schwierig, den zyklischen Verlauf depressiver Störungen abzubilden. Generell ist es schwierig, eine Depression in epidemiologischen Untersuchungen zu erfassen, da die Störung zwar häufig chronisch, oft aber auch episodisch auftritt. Nichtsdestotrotz stellt der DSQ ein effizientes und valides Instrument zur Erfassung der depressiven Symptomatik dar 
[26], das häufig in epidemiologischen Studien eingesetzt wird. Der Follow-up-Zeitraum in der DETECT-Studie mit im Mittel 3,5 Jahren ist im Vergleich zu anderen großen epidemiologischen Längsschnittuntersuchungen eher kurz.

\section{Fazit}

Die Ergebnisse dieser Analysen sprechen dafür, dass inzidente depressive Symptome vor allem mit der Insulinbehandlung und schwereren T2D-Verläufen assoziiert sind. Bei der Entstehung eines T2D scheinen depressive Symptome eher eine vermittelnde Rolle zu spielen. Beide Erkrankungen sind mit einem hohen Mortalitätsrisiko assoziiert, vor allem, wenn sie zusammen auftreten. Auch die aktuelle Fassung der DDG-Praxisleitlinie „Psychosoziales und Diabetes mellitus“ [50] beschriebt den negativen Einfluss depressiver Symptome auf den Verlauf einer T2D-Erkrankung und auf das Selbstmanagement der Patienten. Daher wird den behandelnden Ärzten empfohlen, im Arzt-Patienten-Gespräch auf depressive Symptome zu achten und zur Abklärung gegebenenfalls Screening-Fragebögen einzusetzen. Beim Vorliegen einer depressiven Symptomatik wird je nach Schweregrad auf verschiedene Therapieoptionen verwiesen: psychosomatische Basisversorgung durch den Hausarzt, Einleitung einer Psychotherapie, Verabreichung von Antidepressiva, fachärztliche psychiatrische Mitbehandlung vor allem bei Suizidalität, fachärztliche diabetische Mitbehandlung bei Entgleisung der Stoffwechselparameter. 
Tab. 1 Charakteristika der DETECT-Patienten ohne T2D zur Baseline kategorisiert nach

ihrer depressiven Symptomatik

\begin{tabular}{|c|c|c|c|c|c|}
\hline & \multicolumn{2}{|c|}{$\begin{array}{l}\text { Patienten mit einem } \\
\text { DSQ-Score }<8(N=4759)\end{array}$} & \multicolumn{2}{|c|}{$\begin{array}{l}\text { Patienten mit einem } \\
\text { DSQ-Score } 28 \text { ( } N=661)\end{array}$} & \multirow[t]{2}{*}{$\begin{array}{l}\text { p- } \\
\text { Wert }\end{array}$} \\
\hline & N/MW & $\% / 5 D$ & N/MW & \%/SD & \\
\hline Alter, MW/SD, in Jahren & 55,9 & 14,2 & 57,1 & 14,0 & 0,042 \\
\hline \multicolumn{6}{|l|}{ Geschlecht, N/\% } \\
\hline Männer & 2894 & 61,7 & 448 & 68,4 & \multirow[t]{2}{*}{0,000} \\
\hline Fraven & 1865 & 38,3 & 213 & 31,6 & \\
\hline Schuljahre, MW/SD & 10,2 & 2,2 & 9,8 & 2,0 & 0,000 \\
\hline \multicolumn{6}{|l|}{ Berufstätigkeit } \\
\hline Berufstätig & 2223 & 49,7 & 224 & 35,7 & \multirow[t]{4}{*}{0,000} \\
\hline Hausfrau & 225 & 48 & 64 & 98 & \\
\hline Arbeitslos & 366 & 777 & 57 & 8,3 & \\
\hline Rentner & 1826 & 36,1 & 286 & 42,7 & \\
\hline \multicolumn{6}{|l|}{ Familienstand } \\
\hline Ledig & 469 & 10,5 & 61 & 9,8 & \multirow[t]{3}{*}{0,000} \\
\hline Verheiratet & 3420 & 72,6 & 430 & 64,8 & \\
\hline Geschieden/verwitwet & 827 & 17,0 & 164 & 25,5 & \\
\hline \multicolumn{6}{|l|}{ Rauchstatus, N/\% } \\
\hline Nie & 2592 & 56,2 & 321 & 50,0 & \multirow[t]{3}{*}{0,000} \\
\hline Früher & 1059 & 22,5 & 141 & 21,6 & \\
\hline Aktuell & 964 & 21,3 & 176 & 28,4 & \\
\hline $\begin{array}{l}\text { Probleme, sich gesund zu emähren, } \\
\text { N/\% }\end{array}$ & 1066 & 24,4 & 240 & 39,3 & 0,000 \\
\hline Drug Compliance, MW/SD & 68,0 & 21,0 & 58,4 & 20,0 & 0,000 \\
\hline Behavioral Compliance, MW/SD & 62,2 & 21,3 & 57,1 & 19,6 & 0,000 \\
\hline $\begin{array}{l}\text { Körperliche Bewegung, } \mathrm{N} / \%,>2 \mathrm{~h} / \\
\text { Woche }\end{array}$ & 3218 & 70,5 & 344 & 56,0 & 0,000 \\
\hline \multicolumn{6}{|l|}{ Blutdruck, MW(SD), mmHg } \\
\hline Systolisch & 131,0 & 17,9 & 130,6 & 18,5 & 0,603 \\
\hline Diastolisch & 80,0 & 9,9 & 79,5 & 10,2 & 0,233 \\
\hline Taillenumfang, (WC) MW/SD, cm & 92,7 & 14,1 & 94,5 & 15,5 & 0,004 \\
\hline Insulin, MW/SD, mlU/I & 106 & 14,2 & 11,2 & 16,0 & 0,406 \\
\hline Cholesterin, MW/SD, mg/dl & 224,9 & 41,8 & 225,3 & 46,4 & 0,792 \\
\hline LDL & 128,6 & 33,9 & 127,4 & 34,8 & 0,409 \\
\hline $\mathrm{HDL}$ & 56,1 & 18,7 & 56,2 & 19,6 & 0,869 \\
\hline Triglyzeride, MW(SD), mg/dl & 144,9 & 105,9 & 151,3 & 120,5 & 0,152 \\
\hline C-reaktives Protein, Median/QR, mg// & 1,9 & $0.9-4.2$ & 2,2 & $1,1-4,9$ & 0,003 \\
\hline Inzidente T2D-Falle & 320 & 6,2 & 53 & 7,7 & - \\
\hline Personenjahre & 15289,7 & & 2073,3 & & - \\
\hline
\end{tabular}

DSQ Deptession Screening Questionnare, MW Mittelwert, SD Standardabwelchung, IQR Interquartilsabstand. LDL Low Density Lipoprotein, HDL High Density Lipoprotein.

Tab. 2 Hazard-Ratios für inzidenten T2D nach Baseline-Depressivitäts-Status

(kategorial und dimensional)

\begin{tabular}{|llll|}
\hline Modell & HR $(95 \%$ KI), p-Wert & \\
& & DSQ 28 vs. DSQ $<8$ & Dimensional (punktweiser Anstieg) \\
\hline 1 & Unadjustiert & 1,$24 ; 0,93-1,66 ; 0,140$ & 1,$03 ; 1,01-1,06 ; 0,013$ \\
\hline 2 & Basis & 1,$20 ; 0,90-1,61 ; 0,221$ & 1,$03 ; 1,00-1,06 ; 0,036$ \\
\hline 3 & Taillenumfang & 1,$10 ; 0,82-1,47 ; 0,517$ & 1,$02 ; 1,00-1,05 ; 0,089$ \\
\hline 4 & Metabolisch & 1,$16 ; 0,87-1,55 ; 0,319$ & 1,$03 ; 1,00-1,06 ; 0,033$ \\
\hline 5 & Inflammatonisch & 1,$21 ; 0,90-1,61 ; 0,205$ & 1,$03 ; 1,00-1,06 ; 0,026$ \\
\hline 6 & Soziö̈konomisch & 1,$13 ; 0,85-1,52 ; 0,397$ & 1,$02 ; 0,99-1,05 ; 0,237$ \\
\hline 7 & Lebensstil & 1,$24 ; 0,93-1,66 ; 0,149$ & 1,$03 ; 1,00-1,06 ; 0,025$ \\
\hline 8 & Compliance & 1,$22 ; 0,88-1,70 ; 0,234$ & 1,$03 ; 1,00-1,06 ; 0,081$ \\
\hline 9 & Voll adjustiert & 1,$20 ; 0,86-1,67 ; 0,288$ & 1,$03 ; 1,00-1,06 ; 0,094$ \\
\hline
\end{tabular}

HR Hazard-Ratio, KI Konfidenzinterval, DSQ Depression Screening Questionnale.

Modelle 1. unad Justiert; 2. ad ustiert naxh Alter, Geschlecht und Vertellung der Hausărzte im Bundespebiet:

3. Modell 2 + Tal lenumfang; 4. Modell 3 + Tnglyzenide, HDL, Blutdruck (systolsch, diastolschi), Insulin; 5. Mo-

dell 3 + Creakttves Protein; 6 . Model 3 + Schulahre + Berufsstand; 7. Model 3 + Rauchstatus, korperilche

Ahthitat, Emahrungspoobleme; 8. Modell 3 + Compliance; 9. adjustlert nach alen Kriterien aus alen Modellen. 
Tab. 3 Charakteristika von Patienten ohne depressive Symptomatik zur Baseline nach Diabetesstatus

\begin{tabular}{|c|c|c|c|c|c|c|c|c|c|c|c|}
\hline & \multirow{2}{*}{\multicolumn{2}{|c|}{$\begin{array}{l}\text { Nūchternblutzucker } \\
\text { Normal }(<100 \mathrm{mg} / \mathrm{dl}) \\
\mathrm{N}=3353(63,5 \%)\end{array}$}} & \multicolumn{4}{|c|}{ Typ-2-Diabetes } & \multirow{2}{*}{\multicolumn{2}{|c|}{$\begin{array}{l}\text { Oral behandelt } \\
\mathrm{N}=420(7,9 \%)\end{array}$}} & \multirow{2}{*}{\multicolumn{2}{|c|}{$\begin{array}{l}\text { Insulin/kombi- } \\
\text { niert behandelt } \\
\mathrm{N}=242(4,6 \%)\end{array}$}} & \multirow{3}{*}{$\begin{array}{l}\text { p- } \\
\text { Wert }\end{array}$} \\
\hline & & & \multicolumn{2}{|c|}{$\begin{array}{l}\text { Erhōht (100- } \\
125 \mathrm{mg} / \mathrm{dl}) \\
\mathrm{N}=784(14,9 \%)\end{array}$} & \multicolumn{2}{|c|}{$\begin{array}{l}\text { Medikamentös } \\
\text { unbehandelt } \\
\mathrm{N}=482(9,1 \%)\end{array}$} & & & & & \\
\hline & N/MW & $\% / S D$ & N/MW & $\% / S D$ & N/MW & $\% / S D$ & N/MW & $\% / S D$ & $\mathrm{~N} / \mathrm{MW}$ & $\% / S D$ & \\
\hline Alter, MW/SD, in lahren & 55,2 & 14,2 & 61,0 & 12,4 & 58,6 & 13,3 & 66,4 & 10,1 & 64,8 & 11,0 & 0,000 \\
\hline \multicolumn{12}{|l|}{ Geschlecht, N/\% } \\
\hline Männer & 1214 & 35,1 & 394 & 50,7 & 226 & 45,0 & 235 & 56,5 & 109 & 45,4 & \multirow[t]{2}{*}{0,000} \\
\hline Frauen & 2139 & 64,9 & 390 & 49,3 & 256 & 55,0 & 185 & 43,5 & 133 & 54,6 & \\
\hline Schuljahre, MW/SD & 10,3 & 2,1 & 9,7 & 2,1 & 9,9 & 2,0 & 9,2 & 1,9 & 92 & 177 & 0,000 \\
\hline \multicolumn{12}{|l|}{ Berufstätigkeit } \\
\hline Berufstätig & 1616 & 51,4 & 282 & 38,4 & 200 & 45,7 & 64 & 15,9 & 41 & 17,2 & \multirow[t]{4}{*}{0,000} \\
\hline Hausfrau & 152 & 4,5 & 30 & 4,1 & 23 & 5,0 & 19 & 4,4 & 14 & 6,0 & \\
\hline Arbeitslos & 264 & 7,9 & 43 & 5,5 & 28 & 5,9 & 22 & 5,4 & 14 & 6,0 & \\
\hline Rentner & 1235 & 34,5 & 418 & 51,5 & 219 & 41,6 & 308 & 73,8 & 169 & 69,6 & \\
\hline \multicolumn{12}{|l|}{ Familienstand } \\
\hline Ledig & 338 & 10,6 & 46 & 6,5 & 30 & 6,8 & 24 & 6,0 & 11 & 4,5 & \multirow[t]{3}{*}{0,000} \\
\hline Verheiratet & 2434 & 73,3 & 584 & 75,5 & 349 & 73,6 & 289 & 70,5 & 148 & 61,8 & \\
\hline Geschieden/verwitwet & 552 & 16,1 & 145 & 18,0 & 96 & 19,6 & 99 & 23,5 & 79 & 33,7 & \\
\hline \multicolumn{12}{|l|}{ Rauchstatus, N/\% } \\
\hline Nie & 1879 & 57,4 & 416 & 54,0 & 244 & 55,3 & 219 & 54,3 & 138 & 59,2 & \multirow[t]{3}{*}{0,000} \\
\hline Früher & 736 & 22,1 & 202 & 26,7 & 118 & 25,1 & 136 & 332 & 70 & 30,1 & \\
\hline Aktuell & 659 & 20,5 & 142 & 19,3 & 84 & 19,6 & 51 & 12,5 & 25 & 10,7 & \\
\hline Emährungsprobleme, $N / \%$ & 725 & 23,4 & 172 & 24,6 & 119 & 273 & 101 & 27,4 & 50 & 23,8 & 0,144 \\
\hline Medikamenten-Compliance, MW/SD & 57,5 & 27,1 & 47,1 & 25,9 & 49,2 & 248 & 24,1 & 9,5 & 26,0 & 12,7 & 0,000 \\
\hline Behavioral Compliance, MW/SD & 59,4 & 20,9 & 50,9 & 19,1 & 51,7 & 19,4 & 34,2 & 11,0 & 35,4 & 12,5 & 0,000 \\
\hline Körperliche Bewegung, N/\%, >2 h/Wo & 2315 & 71,5 & 534 & 72,5 & 312 & 68,2 & 267 & 69,3 & 145 & 63,5 & 0,064 \\
\hline Hypertonie, N/\% & 1073 & 27,8 & 393 & 46,0 & 205 & 36,5 & 313 & 70,9 & 180 & 72,2 & 0,000 \\
\hline Taillenumfang, MW/SD, cm & 91,4 & 13,9 & 98,2 & 13,4 & 96,9 & 14,4 & 103,8 & 13,3 & 103,3 & 14,5 & 0,000 \\
\hline Insulin, MW/SD, mlU/ & 7,5 & 7,2 & 16,5 & 17,7 & 23,8 & 30,2 & 15,5 & 168 & 33,7 & 80,7 & 0,000 \\
\hline $\mathrm{HbAlc}, \mathrm{MW} / \mathrm{SD}, \%$ & 5,2 & 0,3 & 5,6 & 0,4 & 5,7 & 0,9 & 6,7 & 1,1 & 7,2 & 12 & 0,000 \\
\hline Cholesterin, MW/SD, mg/dl & 224,5 & 40,8 & 225,4 & 44,4 & 222,5 & 44,2 & 223,4 & 47,3 & 213,0 & 43,7 & 0,401 \\
\hline HDL-Cholesterin, MW/SD, mg/dl & 57,2 & 18,5 & 51,8 & 17,8 & 51,9 & 17,8 & 47,8 & 17,3 & 49,3 & 19,2 & 0,000 \\
\hline Triglyzeride, Median/QR, mg/dl & 137,4 & 99,3 & 169,7 & 115,7 & 170,5 & 136,6 & 217,0 & 266,7 & 177,3 & 120,2 & 0,000 \\
\hline Inzidente depressive Symptome & 342 & 10,1 & 89 & 10,8 & 54 & 11,2 & 46 & 11,0 & 39 & 16,2 & \\
\hline Personenjahre & \multicolumn{2}{|l|}{11154,0} & \multicolumn{2}{|l|}{2587,4} & \multicolumn{2}{|l|}{1550,6} & \multicolumn{2}{|l|}{1423,2} & \multicolumn{2}{|l|}{816,1} & \\
\hline
\end{tabular}

\begin{tabular}{|c|c|c|c|c|c|}
\hline \multirow[t]{2}{*}{ Modell } & \multicolumn{5}{|l|}{ HR (95\% Kl); p-Wert } \\
\hline & $\begin{array}{l}\text { Normaler Nüchtern- } \\
\text { blutzucker (Referenz) }\end{array}$ & $\begin{array}{l}\text { Erhöhter Nūchtern- } \\
\text { blutzudker }\end{array}$ & $\begin{array}{l}\text { Unbehandelter Typ- } \\
\text { 2-Diabetes }\end{array}$ & $\begin{array}{l}\text { Oral behandelter Typ- } \\
\text { 2-Diabetes }\end{array}$ & $\begin{array}{l}\text { Mit Insulin/kombiniert be- } \\
\text { handelter Typ-2-Diabetes }\end{array}$ \\
\hline 1 Unadjustiert & $1, \infty$ & 1,$12 ; 0,89-1,42 ; 0,336$ & 1,$14 ; 0,86-, 52 ; 0,367$ & 1,$05 ; 0,77-1,43 ; 0,752$ & 1,$57 ; 1,12-2,18 ; 0,008$ \\
\hline 2 Basis & 1,00 & $1,09,0,86-1,38 ; 0,488$ & 1,$14 ; 0,85-1,52 ; 0,380$ & 0,$97 ; 0,71-1,33 ; 0,858$ & 1,$44 ; 1,03-2,02 ; 0,034$ \\
\hline Taillenumfang & 1,00 & 1,$08 ; 0,85-1,38 ; 0,507$ & 1,$11 ; 0,83-1,49 ; 0,466$ & 0,$99 ; 0,72-1,37 ; 0,968$ & 1,$48 ; 1,05-2,09 ; 0,024$ \\
\hline 4 Sozioökonomisch & 1,00 & 1,$06 ; 0,84-1,33 ; 0,649$ & $1,10,0,82-1,46 ; 0,524$ & 0,$94 ; 0,69-1,28 ; 0,690$ & 1,$39 ; 0,99-1,93 ; 0,055$ \\
\hline 5 Lebensstil & 1,00 & $1,09,0,86-1,38 ; 0,467$ & 1,$12 ; 0,84-1,50 ; 0,427$ & 1,$03 ; 0,75-1,40 ; 0,863$ & 1,$56 ; 1,12-2,18 ; 0,009$ \\
\hline 6 Lipide, HT, HbAlc & 1,00 & 1,$05 ; 0,83-1,33 ; 0,669$ & $1,09,0,82-1,46 ; 0,539$ & 0,$91 ; 0,66-1,26 ; 0,584$ & 1,$37 ; 0,97-1,92 ; 0,071$ \\
\hline Compliance & 1,00 & 1,$03 ; 0,78-1,35 ; 0,854$ & 1,$02 ; 0,73-1,43 ; 0,900$ & 0,$58 ; 0,36-0,96 ; 0,032$ & 1,$58 ; 1,06-2,36 ; 0,026$ \\
\hline 8 Voll adjustiert & $1, \infty$ & 0,$99 ; 0,75-1,31 ; 0,961$ & 1,$04 ; 0,73-1,47 ; 0,843$ & 0,$62 ; 0,35-1,07 ; 0,088$ & 1,$71 ; 1,03-2,83 ; 0,037$ \\
\hline \multicolumn{6}{|c|}{ 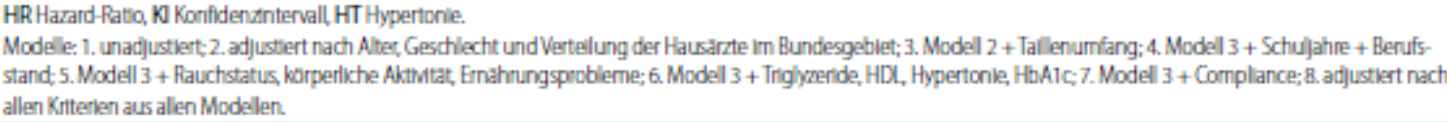 } \\
\hline
\end{tabular}




\begin{tabular}{|c|c|c|c|c|c|c|c|c|c|}
\hline & \multicolumn{2}{|c|}{$\begin{array}{l}\text { Kein T2D und keine depressive } \\
\text { Symptomatik zu TO (Referenz) }\end{array}$} & \multicolumn{2}{|c|}{$\begin{array}{l}\text { Nur depressive Sym- } \\
\text { ptomatik zu T0 }\end{array}$} & \multicolumn{2}{|c|}{$\begin{array}{l}\text { Nur Typ-2-Dia- } \\
\text { betes zu T0 }\end{array}$} & \multicolumn{2}{|c|}{$\begin{array}{l}\text { T2D und depressive } \\
\text { Symptomatikzu T0 }\end{array}$} & \multirow[t]{2}{*}{$\begin{array}{l}\text { p- } \\
\text { Wert }\end{array}$} \\
\hline & N/MW & \%/SD & N/MW & \%/SD & $\mathrm{N} / \mathrm{MW}$ & $\% / S D$ & N/MW & \%/SD & \\
\hline Alter, MW/SD, in Jahren & 55,9 & 14,2 & 57,1 & 14,0 & 67,0 & 9,6 & 64,6 & 11,5 & 0,000 \\
\hline \multicolumn{10}{|l|}{ Geschlecht, $N / \%$} \\
\hline Männer & 2894 & 61,7 & 448 & 68,4 & 465 & 47,2 & 87 & 53,6 & \multirow[t]{2}{*}{0,000} \\
\hline Frauen & 1865 & 38,3 & 213 & 31,6 & 511 & 52,8 & 78 & 46,4 & \\
\hline Schuljahre, MW/SD & 10,2 & 2,2 & 9,8 & 2,0 & 9,2 & 1,8 & 9,2 & 1,9 & 0,000 \\
\hline \multicolumn{10}{|l|}{ Berufstätigkeit } \\
\hline Berufstätig & 2223 & 49,7 & 224 & 35,7 & 142 & 15,0 & 18 & 10,7 & \multirow[t]{4}{*}{0,000} \\
\hline Hausfrau & 225 & 4,8 & 64 & 9,8 & 40 & 4,2 & 16 & 10,3 & \\
\hline Arbeitslos & 366 & 7,7 & 57 & 8,3 & 48 & 5,0 & 12 & 7,3 & \\
\hline Rentner & 1826 & 36,1 & 286 & 42,7 & 732 & 75,2 & 113 & 69,1 & \\
\hline \multicolumn{10}{|l|}{ Familienstand } \\
\hline Ledig & 469 & 10,5 & 61 & 9,8 & 41 & 4,3 & 10 & 5,9 & \multirow[t]{3}{*}{0,000} \\
\hline Verheiratet & 3420 & 72,6 & 430 & 64,8 & 658 & 68,9 & 107 & 66,3 & \\
\hline Geschieden/verwitwet & 827 & 17,0 & 164 & 25,5 & 259 & 268 & 46 & 27,8 & \\
\hline \multicolumn{10}{|l|}{ Rauchstatus, N/\% } \\
\hline Nie & 2592 & 56,2 & 321 & 50,0 & 522 & 55,6 & 88 & 56,9 & \multirow[t]{3}{*}{0,000} \\
\hline Früher & 1059 & 22,5 & 141 & 21,6 & 301 & 32,1 & 43 & 27,5 & \\
\hline Aktuell & 964 & 21,3 & 176 & 28,4 & 114 & 12,3 & 25 & 15,7 & \\
\hline Emährungsprobleme, N/\% & 1066 & 24,4 & 240 & 39,3 & 209 & 24,7 & 61 & 42,9 & 0,000 \\
\hline Medikamenten-Compliance, MW/SD & 68,0 & 21,0 & 58,4 & 20,0 & 45,4 & 13,4 & 44,2 & 15,0 & 0,000 \\
\hline Behavioral Compliance, MW/SD & 62,2 & 21,3 & 57,1 & 19,6 & 37,8 & 13,4 & 31,3 & 14,0 & 0,000 \\
\hline Körperliche Bewegung, $\mathrm{N} / \%,>2 \mathrm{~h} / \mathrm{Wb}$ & 3218 & 70,5 & 344 & 56,0 & 612 & 687 & 70 & 49,7 & 0,000 \\
\hline Hypertonie, N/\% & 2636 & 51,5 & 404 & 57,4 & 901 & 91,2 & 155 & 93,0 & 0,000 \\
\hline Taillenumfang, MW/SD, cm & 92,7 & 14,1 & 94,5 & 15,5 & 103,1 & 13,0 & 105,4 & 14,6 & 0,000 \\
\hline Insulin, MW/SD, mIlU/ & 10,5 & 14,4 & 12,8 & 20,2 & 20,1 & 43,9 & 21,9 & 21,5 & 0,000 \\
\hline Cholesterin, MW/SD, mg/dl & 224,9 & 41,8 & 225,3 & 46,4 & 218,6 & 45,6 & 214,5 & 56,6 & 0,000 \\
\hline LDL & 128,6 & 33,9 & 127,4 & 34,8 & 124,4 & 33,1 & 119,6 & 36,7 & 0,000 \\
\hline HDL & 56,1 & 18,7 & 56,2 & 19,6 & 48,2 & 16,9 & 44,9 & 17,7 & 0,000 \\
\hline Triglyzeride, Median/QR, mg/dl & 144,9 & 105,9 & 151,3 & 120,5 & 197,4 & 205,0 & 211,6 & 211,9 & 0,000 \\
\hline All cause Mortalität & 123 & 2,5 & 41 & 6,0 & 76 & 7,8 & 16 & 9,8 & \\
\hline Personenjahre & 15289,7 & & 2073,3 & & 3266,7 & & 506,0 & & \\
\hline
\end{tabular}

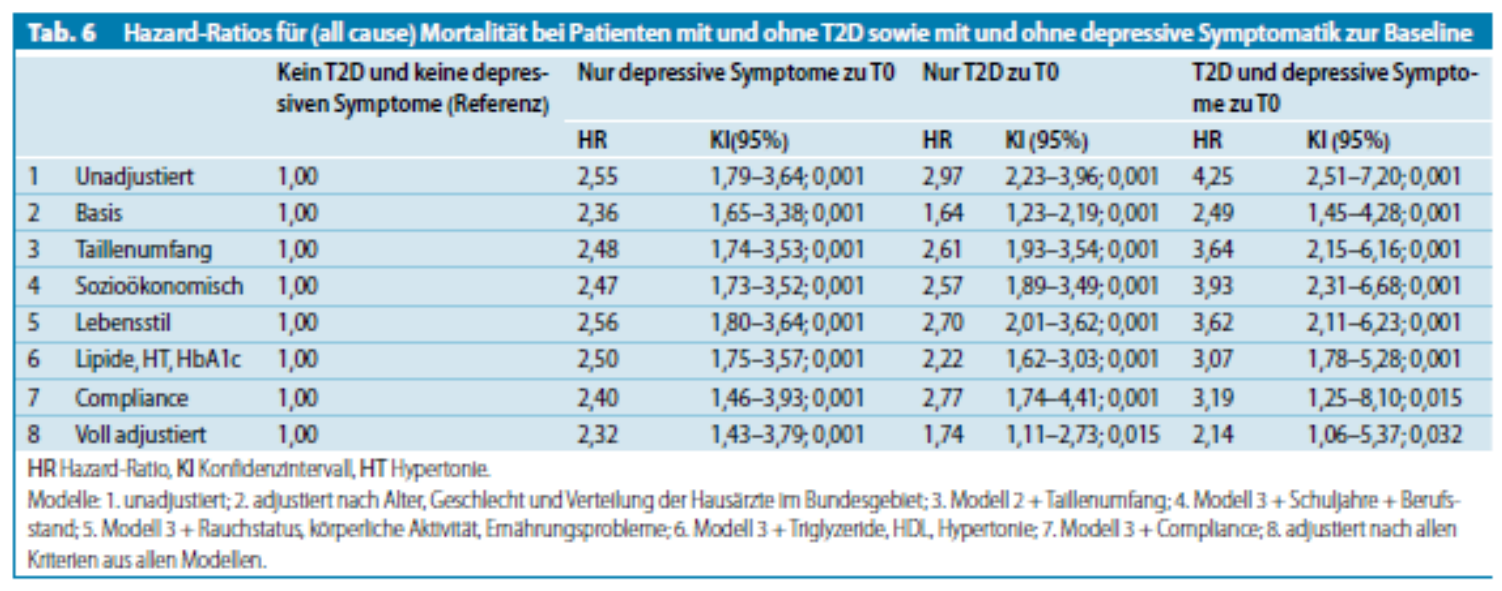

\section{Hinweis}

DETECT (Diabetes Cardiovascular Risk-Evaluation: Targets and Essential Data for Commitment of Treatment) is a cross-sectional and prospective longitudinal, nationwide clinical epidemiological study. DETECT is supported by an unrestricted educational grant of Pfizer GmbH, Karlsruhe, Germany. Principal investigator: Prof. Dr. H.-U. Wittchen; Staff members: Dipl.-Psych. L. Pieper, Dipl.-Math. J. Klotsche, Dr. T. Eichler, Dr. H. Glaesmer, E. Katze. Steering Committee: Prof. Dr. H. Lehnert (Lübeck), Prof. Dr. G.K. Stalla (München), Prof. Dr. A.M. Zeiher (Frankfurt); Advisory Board: Prof. Dr. W. März (Heidelberg/Graz), 
Prof. Dr. S. Silber (München), Prof. Dr. Dr. U. Koch (Hamburg), Prof. Dr. D. Pittrow (München/Dresden), Prof. Dr. M. Wehling (Mannheim), Dr. D. Leistner (Frankfurt), Dr. H.J. Schneider (München), Dr. C. Sievers (München).

\section{Korrespondenzadresse}

Dipl.-Psych. L. Pieper

Institut für Klinische Psychologie und

Psychotherapie, Technische Universität Dresden

Chemnitzer Str. 46, 01187 Dresden

pieper@psychologie.tu-dresden.de

\section{Interessenkonflikt}

Der korrespondierende Autor gibt an, dass kein Interessenkonflikt besteht.

\section{Literatur}

1. Talbot F, Nouwen A (2000) A review of the relationship between depression and diabetes in adults: Is there a link? Diabetes Care 23:1556-1562

2. Anderson RJ, Freedland KE, Clouse RE, Lustman PJ (2001) The prevalence of comorbid depression in adults with diabetes: a meta-analysis. Diabetes Care 24:1069-1078

3. Golden SH, Lazo M, Carnethon M et al (2008) Examining a bidirectional association between depressive symptoms and diabetes. JAMA 299:2751-2759

4. Eaton WW, Armenian H, Gallo J et al (1996) Depression and risk for onset of type II diabetes: a prospective population-based study. Diabetes Care 19:1097-1102

5. Carnethon MR, Kinder LS, Fair JM et al (2003) Symptoms of depression as a risk factor for incident diabetes: findings from the National and Nutrition Examination Epidemiologic follow-up study, 1971-1992. Am J Epidemiol 158:416-423

6. Arroyo C, Hu FB, Ryan LM et al (2004) Depressive symptoms and risk of type 2 diabetes in women. Diabetes Care 27:129-133

7. Knoll MJ, Twisk JW, Beekman AT et al (2006) Depression as a risk factor for the onset of type 2 diabetes mellitus: a meta-analysis. Diabetologia 49:837-845

8. Mezuk B, Eaton W, Albrecht S et al (2008) Depression and type 2 diabetes over the Lifespan. Diabetes Care 31:2383-2390

9. Everson-Rose SA, Meyer PM, Powell LH et al (2004) Depressive symptoms, insulin resistance, and risk of diabetes in woman at midlife. Diabetes Care 27:2856-2862

10. Golden SH (2007) A review of the evidence for a neuroendocrine link between stress, depression and diabetes mellitus. Curr Diabetes Rev 3:252-259

11. Ford DE, Erlinger TP (2004) Depression and C-reactive protein in US-adults: data from the Third National Health and Nutrition Examination Survey. Arch Intern Med 164:10101014

12. Duncan BB, Schmidt MI, Pankow JS et al (2003) Low grade systemic inflammation and the development of type 2 diabetes: the atherosclerosis risk in communities study. Diabetes 52:1799-1805

13. Saydah SH, Brancati FL, Golden SH et al (2003) Depressive symptoms and the risk of type 2 diabetes mellitus in a US sample. Diabetes Metab Res Rev 19:202-208

14. Hermanns N, Kulzer B, Krichbaum M et al (2005) Affective and anxiety disorders in a German sample of diabetic patients: prevalence, comorbidity and risk factors. Diabet Med 22:293-300 
15. DeJonge P, Roy JF, Saz P et al (2006) Prevalent and incident depression in communitydwelling elderly persons with diabetes mellitus: results from the ZARADEMP project.

Diabetologia 49:2627-2633

16. Maraldi C, Volpato S, Penninx BW et al (2007) Diabetes mellitus, glycemic control, and incident depressive symptoms among 70- to 79-year-old persons: the health, aging, and body composition study. Arch Intern Med 167:1137-1144

17. Lawlor DA, Hart CL, Hole DJ et al (2007) Body mass index in middle life and future risk of hospital admission for psychose or depression: findings from the Renfrew/Paisley study.

Psychol Med 37:1151-1161

18. Lawlor DA, Smith GD, Ebrahim S (2003) Association of insulin resistance with depression: cross sectional findings from the British Woman's Heart and Health study. BMJ 327:1383-1384

19. Payrot $M$ et al (1997) Levels and risks of depression and anxiety symptomatology among diabetic adults. Diabetes Care 20:585-590

20. Engum A, Mykletun A, Kristian K et al (2005) Depression and diabetes. A large population-based study of sociodemographic, lifestyle, and clinical factors associated with depression in type 1 and type 2 diabetes. Diabetes Care 28:1904-1909

21. Black SA, Markides KS, Ray LA (2003) Depression predicts increased incidence of adverse health outcomes in older Mexican Americans with type 2 diabetes. Diabetes Care 26:2822-2828

22. Egede LE, Neitert PJ, Zheng D (2005) Depression and all-cause and coronary heart disease mortality among adults with and without diabetes. Diabetes Care 28:1339-1345 23. Wittchen HU, Glaesmer H, März W et al (2005) Cardiovascular risk factors in primary care: methods and baseline prevalence rates - the DETECT program. Curr Med Res Opin 21:619-629

24. Pieper L, Wittchen HU, Glaesmer H et al (2005) Kardiovaskuläre

Hochrisikokonstellationen in der primärärztlichen Versorgung: DETECT-Studie 2003.

Bundesgesundheitsbl Gesundheitsforsch Gesundheitsschutz 12:1374-1382

25. Winter S, Wittchen HU, Höfler M et al (2000) Design und Methoden der Studie „Depression 2000“. Charakteristik der teilnehmenden Ärzte und Patienten. MMW Fortschr Med 118:11-21

26. Wittchen HU, Winter S, Höfler M et al (2000) Häufigkeit und Erkennensrate von Depression in der hausärztlichen Praxis. MMW Fortschr Med 118:22-30

27. Pittrow D, März W, Zeiher AM et al (2006) Prävalenz, medikamentöse Behandlung und Stoffwechseleinstellung des Diabetes mellitus in der Hausarztpraxis. Med Klin 101:635-644 28. Clayton D, Hills M (1993) Statistical models in epidemiology. Oxford University Press, Oxford 29. Singer JD, Willett JB (1993) It's about time: using discrete-time survival analysis to study duration and the timing of events. J Educ Stat 18:155-195

30. Cox DR, Oakes D (1984) Analysis of survival data. Chapman and Hall, London 31. Royall RM (1986) Model robust confidence intervals using maximum likelihood estimators. Int Stat Rev 54:221-226

32. StataCorp. (2009) Stata Statistical Software. Release 11.0. Stata Corporation, College Station, TX

33. Räikkönen K, Matthews KA, Kuller LH (2007) Depressive symptoms and stressful life events predict metabolic syndrome among middle-aged women. Diabetes Care 30:872-877 34. Kruse J, Schmitz N, Thefeld W (2003) On the association between diabetes and mental disorders in a community sample. Diabetes Care 26:1841-1846

35. Musselman DL, Betan E, Larsen H, Phillips LS (2003) Relationship of depression to diabetes types 1 and 2: epidemiology, biology, and treatment. Biol Psychiatry 54:317-329 36. Egede LE (2004) Effect of depression on work loss and disability bed days in individuals with diabetes. Diabetes Care 27:1751-1753 
37. Groot M de, Anderson R, Freedland KE et al (2001) Association of depression and diabetes complications: a meta-analysis. Psychosom Med 63:619-630

38. Miyaoka Y, Miyaoka H, Motomiya T et al (1997) Impact of sociodemographic and diabetes related characteristics on depressive state among noninsulin-dependent diabetic patients. Psychiatry Clin Neurosci 51:203-206

39. Cohen ST, Welch G, Jacobson AM et al (1997) The association of lifetime psychiatric illness and increased retinopathy in patients with type I diabetes mellitus. Psychosomatics 38:98-108

40. Viinamaki H, Niskanen L, Uusitupa M (1995) Mental wellbeing in people with noninsulin-dependent diabetes. Acta Psychiatr Scand 92:392-397

41. Geringer ES, Perlmuter LC, Stern TA, Nathan DM (1988) Depression and diabetic neuropathy: a complex relationship. J Geriatr Psychiatry Neurol 1:11-15

42. Black SA (1999) Increased health burden associated with comorbid depression in older diabetic Mexican Americans: results from the Hispanic Established Population for the Epidemiologic Study of the Elderly Survey. Diabetes Care 22:56-64

43. Winocour PH, Main CJ, Medlicott G, Anderson DC (1990) A psychometric evaluation of adult patients with type 1 (insulin-dependent) diabetes mellitus: prevalence of psychological dysfunction and relationship to demographic variables, metabolic control and complications. Diabetes Res 14:171-176

44. Lin EHB, Katon W, Von Korff M et al (2004) Relationship of depression and diabetes self-care, medication adherence, and preventive care. Diabetes Care 27:2154-2160 45. Katon W, Cantrell CR, Sokol MC et al (2005) Impact of antidepressant drug Aadherence on comorbid medication use and resource utilization. Arch Intern Med 165:2497-2503 46. Richardson LK, Egede LE, Mueller M et al (2008) Longitudinal effects of depression on glycemic control in veterans with Type 2 diabetes. Gen Hosp Psychiatry 30:509-514 47. Petrak F, Stridde E, Huppertz E et al (2006) Depression and treatment choice in type 2 diabetes patient failing oral antidiabetic treatment. Diabetologia 49:870

48. Goldney RD, Phillips PJ, Fisher LJ et al (2004) Diabetes, eepression and quality of life. A population study. Diabetes Care 27:1066-1070

49. Von Korff M, Ludman E, Katon W et al (2005) Work disability among individuals with diabetes. Diabetes Care 28:1326-1332

50. Kulzer B, Albus C, Herpertz S et al (2009) Psychosoziales und Diabetes mellitus. Diabetologie 4:S150-S156 